\title{
Multiple Functional Motifs Are Required for the Tumor Suppressor Activity of a Constitutively-Active ErbB4 Mutant
}

\author{
Richard M. Gallo ${ }^{1,2 \#}$, Ianthe N. Bryant ${ }^{1 \#}$, Christopher P. Mill ${ }^{1,3}$, Steven Kaverman ${ }^{1}$ and David J. Riese II ${ }^{1,3^{*}}$ \\ ${ }^{1}$ Department of Medicinal Chemistry and Molecular Pharmacology, Purdue University College of Pharmacy \& \\ Purdue University Center for Cancer Research, West Lafayette, IN 47907, USA \\ ${ }^{2}$ Department of Microbiology and Immunology, Indiana University School of Medicine, Indianapolis, IN 46206 \\ USA \\ ${ }^{3}$ Department of Pharmacal Sciences, Auburn University Harrison School of Pharmacy, Auburn, AL 36849-5501 \\ USA
}

${ }^{\star}$ Corresponding author: David J. Riese II, Department of Pharmacal Sciences, Harrison School of Pharmacy, Auburn University, Auburn, AL 36849-5501, USA. Email: driese@auburn.edu

"Co-first authors: These authors contributed equally to this work.

Received Date: July 22, 2013 Accepted Date: August 20, 2013 Published Date: August 22, 2013

Citation: Richard M. Gallo, et al. (2013) Multiple Functional Motifs Are Required for the Tumor Suppressor Activity of a Constitutively-Active ErbB4 Mutant. J Cancer Res Therap Oncol 1: 1-10

\begin{abstract}
ErbB4 (HER4) is a member of the ErbB family of receptor tyrosine kinases, which includes the Epidermal Growth Factor Receptor (EGFR/ErbB1), ErbB2 (HER2/Neu), and ErbB3 (HER3). Mounting evidence indicates that ErbB4, unlike EGFR or ErbB2, functions as a tumor suppressor in many human malignancies. Previous analyses of the constitutively-dimerized and -active ErbB4 Q646C mutant indicate that ErbB4 kinase activity and phosphorylation of ErbB4 Tyr1056 are both required for the tumor suppressor activity of this mutant in human breast, prostate, and pancreatic cancer cell lines. However, the cytoplasmic region of ErbB4 possesses additional putative functional motifs, and the contributions of these functional motifs to ErbB4 tumor suppressor activity have been largely underexplored. Here we demonstrate that ErbB4 BH3 and LXXLL motifs, which are thought to mediate interactions with $\mathrm{Bcl}$ family proteins and steroid hormone receptors, respectively, are required for the tumor suppressor activity of the ErbB4 Q646C mutant. Furthermore, abrogation of the site of ErbB4 cleavage by gamma-secretase also disrupts the tumor suppressor activity of the ErbB4 Q646C mutant. This last result suggests that ErbB4 cleavage and subcellular trafficking of the ErbB4 cytoplasmic domain may be required for the tumor suppressor activity of the ErbB4 Q646C mutant. Indeed, here we demonstrate that mutants that disrupt ErbB4 kinase activity, ErbB4 phosphorylation at Tyr1056, or ErbB4 cleavage by gamma-secretase also disrupt ErbB4 trafficking away from the plasma membrane and to the cytoplasm. This supports a model for ErbB4 function in which ErbB4 tumor suppressor activity is dependent on ErbB4 trafficking away from the plasma membrane and to the cytoplasm, mitochondria, and/or the nucleus.
\end{abstract}

Keywords: ErbB4/HER4; Signal Transduction; Tumor Suppressor; Protein Trafficking

\section{Introduction}

ErbB4 (HER4) is a member of the ErbB family of receptor tyrosine kinases, a family that also includes the Epidermal Growth Factor Receptor (EGFR/HER1), ErbB2 (HER2/Neu), and ErbB3 (HER3). Like other members of the ErbB family, ErbB4 possesses extracellular ligand-binding motifs, a hydrophobic transmembrane domain, and a cytoplasmic tyrosine kinase domain (Figure 1). Ligands for ErbB4 are members of the Epidermal Growth Factor (EGF) family of peptide growth factors. Binding of one of these ligands to ErbB4 causes receptor dimerization, phosphorylation of multiple cytoplasmic

C2013 The Authors. Published by the JScholar under the terms of the Creative Commons Attribution License http://creativecommons.org/licenses/ by $/ 3.0 /$, which permits unrestricted use, provided the original author and source are credited. tyrosine residues of ErbB4, ErbB4 binding to effectors that possess $\mathrm{SH} 2$ or PTB binding motifs, and activation of multiple downstream signaling pathways [1-4].

There are two sites of alternative splicing of the ErbB4 transcript, one in the juxtamembrane (JM) region and one in the cytoplasmic tail (CT) region, giving rise to four distinct ErbB4 isoforms [5, 6]. Relative to the canonical JM-a/CT-a isoform (aka JM-a/Cyt1), the JM-b isoforms possess an alternative short sequence in the extracellular juxtamembrane region of the protein (Figure 1). The CT-b isoforms lack a short sequence in the cytoplasmic region of the protein, distal to the tyrosine kinase domain.

Aside from the ligand-binding, transmembrane, and tyrosine kinase domains, ErbB4 possesses a number of other motifs that may be critical for ErbB4 function (Figure 1). These 


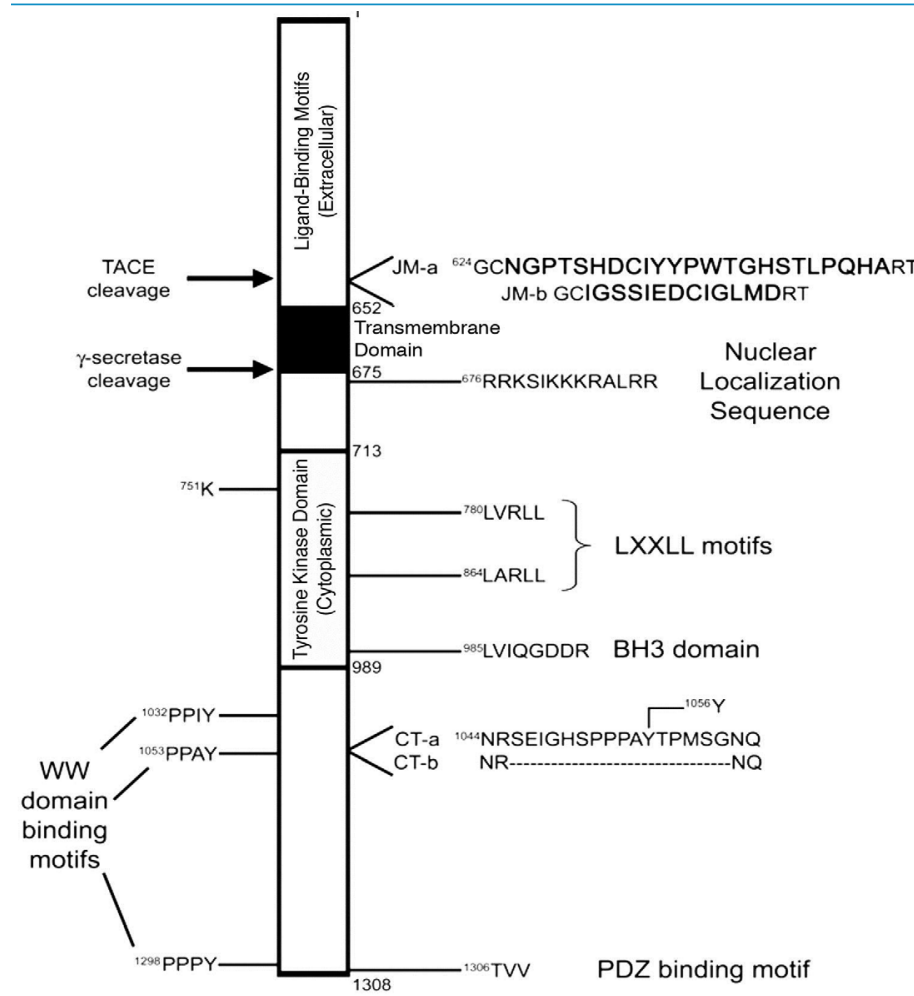

$\begin{array}{ll}\text { Mutations } & \\ \text { Q646C } & \text { Causes constitutive signaling } \\ \text { V673I } & \text { Disrupts putative } \gamma \text {-secretase cleavage site } \\ \text { K751M } & \text { Abrogates tyrosine kinase activity } \\ \text { LL783/4AA } & \text { Targets amino-terminal LXXLL motif } \\ \text { LL867/8AA } & \text { Targets carboxyl-terminal LXXLL motif } \\ \text { L985A } & \text { Targets BH3 domain } \\ \text { D990A } & \text { Targets BH3 domain } \\ \text { Y1056F } & \text { Eliminates site of phosphorylation }\end{array}$

Figure 1: ErbB4 Possesses Multiple Functional Motifs and Mutations Have Been Engineered to Target These Motifs. The organization of ErbB4 is as indicated in this schematic. The extracellular ligand-binding motifs reside in the amino-terminal region upstream of amino acid residue 651 . The singlepass transmembrane domain consists of amino acid residues 652-675. The cytoplasmic tyrosine kinase domain consists of amino acid residues 713-989. The majority of cytoplasmic sites of tyrosine phosphorylation reside in amino acid residues 990-1308, most notably Tyr1056. Additional putative functional motifs include a TACE cleavage site, a gamma-secretase cleavage site, two LXXLL (steroid hormone receptor binding) motifs, a BH3 domain, three WW domain binding motifs, and a PDZ domain binding motif. Mutations that disrupt these motifs are noted. Finally, note the two locations of alternative transcriptional splicing, resulting in a total of four different splicing isoforms.

include sites for cleavage by Tumor Necrosis Factor AlphaConverting Enzyme (TACE) and gamma-secretase [5, 7-9], a nuclear localization sequence [10], LXXLL motifs (which may enable interactions with nuclear hormone receptors) [11-13], a BH3 domain [14] (which enables binding to Bcl family proteins), motifs for binding to WW domains $[15,16]$, and a motif for binding to PDZ domains $[17,18]$. The JM-b isoforms do not contain the canonical site for cleavage by TACE; the CTbisoforms lack a putative site of phosphorylation (Tyr1056) and a motif for binding to WW domains. EGFR and ErbB2 are potent oncoproteins whose aberrant signaling is associated with a number of human malignancies [2-4, 19-23]. In contrast, there is much evidence from clinical studies and laboratory investigations indicating that ErbB4 possesses tumor suppressor ivities [14, 24-39]. We have previously generated the ErbB4 Q646C mutant, which displays ligand-independent dimerization and tyrosine phosphorylation [40, 41].
This mutant inhibits clonogenic proliferation of several human breast, prostate, and pancreatic tumor cell lines [40, 42, 43]. This tumor suppressor activity requires a functional ErbB4 kinase domain as well as Tyr1056 [42, 44].

Multiple reports indicate that a recombinant ErbB4 cytoplasmic domain functions as a tumor suppressor, suggesting that cleavage of the native ErbB4 protein by Tumor Necrosis Factor alpha Converting Enzyme (TACE) and/or gamma-secretase and release of the ErbB4 intracellular domain (4ICD or s80 fragment) from the plasma membrane are required for ErbB4 tumor suppressor activity [35, 45-50]. However, heretofore we have not established whether the other ErbB4 functional motifs found in the cytoplasmic region of ErbB4 are required for the tumor suppressor activity of the ErbB4 Q646C mutant. Thus, here we use ErbB4 mutants to demonstrate that additional ErbB4 motifs are required for the ErbB4 Q646C mutant to inhibit clonogenic proliferation of breast and prostate tumor cell lines. We also present data that suggest some of the ErbB4 mutants defective for tumor suppressor activity also exhibit aberrant subcellular localization. Thus, we postulate that the tumor suppressor activity of the constitutively-active ErbB4 mutant may be regulated by subcellular trafficking.

\section{Materials and Methods Cell lines and cell culture}

Mouse C127 fibroblasts and the $\Psi 2$ and PA317 recombinant retrovirus packaging cell lines are generous gifts of Dr. Daniel DiMaio (Yale University, New Haven, Connecticut, USA). These cells were cultured essentially as described [41, 43, 51]. The following cell lines were obtained from the American Type Culture Collection (ATCC): PC-3 and DU-145 human prostate tumor cell lines, the MCF7 human mammary tumor cell line and the MCF10A human mammary epithelial cell line. These cell lines were cultured in accordance with ATCC recommendations. Cell culture media and supplements were obtained from GE Biosciences (formerly GIBCO/BRL/Life Technologies). Fetal bovine serum and G418 were obtained from Gemini Bioproducts. Plasticware and Giemsa stain were obtained from Fisher Scientific.

\section{ErbB4 mutants}

Unless otherwise noted, all ErbB4 constructs used in this paper were generated in the context of the recombinant retrovirus expression vector pLXSN-ErbB4 [52], which encodes the canonical 1308-amino acid, human ErbB4 JM-a CT-a isoform. The constitutively-dimerized and -active Q646C ErbB4 mutant has been previously described [40, 41], as have the kinase-deficient K751M ErbB4 mutant and the Y1056F ErbB4 phosphorylation site mutant $[42,44]$. Additional ErbB4 mutants were generated by standard site-directed mutagenesis and subcloning techniques (Figure 1). The sequence of each mutagenic primer is available upon request.

The ErbB4 V673I mutation, in which the Val673 codon is changed to an isoleucine codon, disrupts ErbB4 cleavage by gamma-secretase [35]. The ErbB4 LL783/4AA mutation, in which the Leu783 and Leu784 codons are both simultaneously changed to an alanine codon, is predicted to disrupt ErbB4 binding to nuclear hormone receptors [11-13]. The ErbB4 
LL867/8AA mutation, in which the Leu867 and Leu868 codons are both simultaneously changed to an alanine codon, is also predicted to disrupt ErbB4 binding to steroid hormone receptors. The ErbB4 L985A and D990A mutations, in which the Leu985 and Asp990 codons are each changed to an alanine, are predicted to disrupt ErbB4 binding to Bcl family proteins [53]. ErbB4 expression and tyrosine phosphorylation was detected by immunoprecipitation and immunoblotting as previously described [40, 41, 54].

\section{ErbB4-EGFP fusion constructs}

The ErbB4 Enhanced Green Fluorescent Protein (EGFP) fusion construct, in which the EGFP sequence is added to the carboxyl terminus of the ErbB4, has been previously described [10] and is the generous gift of Dr. Frank Jones (Tulane University, New Orleans, Louisiana, USA). To generate the ErbB4 Q646C EGFP construct, the DraIII-HpaI fragment of this construct, which encodes the carboxyl-terminal portion of ErbB4 and the entire EGFP sequence, was ligated to the DraIII -BamHI fragment of pLXSN-ErbB4-Q646C [41], which encodes the recombinant retroviral sequences and the aminoterminal portion of ErbB4, including the Q646C mutation. We used standard site-directed mutagenesis to add the sequence that encodes Thr-Val-Val to the 3' end of the sequence that encodes the EGFP tag.

\section{Inhibition of clonogenic proliferation by ErbB4}

This assay was performed essentially as described [40, 42-44]. Briefly, all ErbB4 constructs were expressed from the recombinant retroviral expression vector pLXSN, which carries the neomycin resistance gene [55] and is a generous gift of Dr. Daniel DiMaio (Yale University). A high-titer amphotropic retroviral stock was generated from each construct using standard techniques, the $\Psi^{2}$ ecotropic retrovirus packaging cell line, and the PA317 amphotropic retrovirus packaging cell line [56, 57]. The DU-145, PC-3, MCF7, and MCF10A cell lines were infected with these viruses and stably infected cellswere selected using

\begin{tabular}{|l|l|l|l|}
\hline Cell Line & Retrovirus & $\begin{array}{l}\text { Clonogenic Efficiency } \\
\text { (Relative to C127 } \\
\text { Cells) }\end{array}$ & $\begin{array}{l}\text { Inhibition of } \\
\text { Clonogenic- } \\
\text { ity (Relative to } \\
\text { LXSN) }\end{array}$ \\
\hline $\begin{array}{l}\text { MCF7 } \\
(\mathrm{n}=4)\end{array}$ & LXSN & $2.0 \%$ & N/A \\
\cline { 2 - 4 } & ErbB4 & $1.6 \%$ & $15 \pm 2 \%$ \\
\cline { 2 - 4 } & ErbB4 Q646C & $0.04 \%$ & $97 \pm 1 \%$ \\
\cline { 2 - 4 } & $\begin{array}{l}\text { ErbB4 Q646C } \\
\text { K751M }\end{array}$ & $3.3 \%$ & None \\
\cline { 2 - 4 } & $\begin{array}{l}\text { ErbB4 Q646C } \\
\text { V673I }\end{array}$ & $2.5 \%$ & None \\
\cline { 2 - 4 } & $\begin{array}{l}\text { ErbB4 Q646C } \\
\text { LL783/4AA }\end{array}$ & $2.4 \%$ & None \\
\cline { 2 - 4 } & $\begin{array}{l}\text { ErbB4 Q646C } \\
\text { LL867/8AA }\end{array}$ & $0.5 \%$ & $70 \pm 7 \%$ \\
\cline { 2 - 4 } & $\begin{array}{l}\text { ErbB4 Q646C } \\
\text { L985A }\end{array}$ & $2.9 \%$ & None \\
\cline { 2 - 4 } & $\begin{array}{l}\text { ErbB4 Q646C } \\
\text { D990A }\end{array}$ & $1.2 \%$ & $35 \pm 13 \%$ \\
\hline
\end{tabular}

Table 1: The K751M, V673I, LL783/4AA, and L985A mutations markedly disrupt the tumor suppressor activity of the constitutively-active ErbB4 Q646C mutant in the MCF7 human breast tumor cell line.
G418. By comparing the number of drug-resistant colonies that arise from infection with the tester viruses to the number of drug-resistant colonies that arise from infection with the control viruses (typically the pLXSN vector control virus or the virus encoding the kinase-deficient [K751M] ErbB4 Q646C mutant), we assessed the effects of the ErbB4 mutants on clonogenic proliferation of these human prostate and breast cell lines.

\begin{tabular}{|c|c|c|c|}
\hline Cell Line & Retrovirus & $\begin{array}{l}\text { Clonogenic } \\
\text { Efficiency } \\
\text { (Relative to C127 } \\
\text { Cells) }\end{array}$ & $\begin{array}{l}\text { Inhibition of } \\
\text { Clonogenic- } \\
\text { ity (Relative to } \\
\text { LXSN) }\end{array}$ \\
\hline \multirow{5}{*}{$\begin{array}{l}\text { Expt. 1 } \\
\text { MCF10A } \\
(\mathrm{n}=4)\end{array}$} & LXSN & $9.8 \%$ & N/A \\
\hline & ErbB4 & $10.0 \%$ & None \\
\hline & ErbB4 Q646C & $0.8 \%$ & $91 \pm 1 \%$ \\
\hline & $\begin{array}{l}\text { ErbB4 Q646C } \\
\text { K751M }\end{array}$ & $8.5 \%$ & None \\
\hline & $\begin{array}{l}\text { ErbB4 Q646C } \\
\text { V673I }\end{array}$ & $8.5 \%$ & None \\
\hline \multirow{6}{*}{$\begin{array}{l}\text { Expt. } 2 \\
\text { MCF10A } \\
(\mathrm{n}=3)\end{array}$} & LXSN & $4.3 \%$ & N/A \\
\hline & ErbB4 & $6.2 \%$ & None \\
\hline & ErbB4 Q646C & $0.5 \%$ & $87 \pm 2 \%$ \\
\hline & $\begin{array}{l}\text { ErbB4 Q646C } \\
\text { K751M }\end{array}$ & $9.5 \%$ & None \\
\hline & $\begin{array}{l}\text { ErbB4 Q646C } \\
\text { LL783/4AA }\end{array}$ & $7.4 \%$ & None \\
\hline & $\begin{array}{l}\text { ErbB4 Q646C } \\
\text { LL867/8AA }\end{array}$ & $1.8 \%$ & $58 \pm 6 \%$ \\
\hline \multirow{6}{*}{$\begin{array}{l}\text { Expt. } 3 \\
\text { MCF10A } \\
(\mathrm{n}=3)\end{array}$} & LXSN & $5.8 \%$ & N/A \\
\hline & ErbB4 & $7.1 \%$ & None \\
\hline & ErbB4 Q646C & $0.6 \%$ & $88 \pm 3 \%$ \\
\hline & $\begin{array}{l}\text { ErbB4 Q646C } \\
\text { K751M } \\
\end{array}$ & $7.4 \%$ & None \\
\hline & $\begin{array}{l}\text { ErbB4 Q646C } \\
\text { L985A }\end{array}$ & $5.8 \%$ & None \\
\hline & $\begin{array}{l}\text { ErbB4 Q646C } \\
\text { D990A }\end{array}$ & $4.4 \%$ & $28 \pm 13 \%$ \\
\hline
\end{tabular}

Table 2: The K751M, V673I, LL783/4AA, and L985A mutations markedly disrupt the tumor suppressor activity of the constitutively-active ErbB4 Q646C mutant in the MCF10A human breast epithelial cell line.

Our analysis of what we term clonogenic proliferation is formally a combination of plating efficiency and the ability of the seeded cells to proliferate into a visible colony of drug-resistant cells. We have previously demonstrated that the failure of MCF7 cells that have been infected with the pLXSN-ErbB4Q646C retrovirus to form colonies of drug-resistant cells does not appear to be due to a diminished plating efficiency, but rather is a consequence of growth arrest [42]. Consequently, our analysis of what we term clonogenic proliferation is likely to primarily be an analysis of clonogenic proliferation and not of plating efficiency. Analyses of these data were performed as described elsewhere [40]. Briefly, we normalized for differences in virus titer by infecting $\mathrm{C} 127$ fibroblasts in parallel; this permitted us to calculate the efficiency of each virus in causing clonogenic proliferation in the tester cell lines ("clonogenic efficiency") by dividing the titer of a given viral stock in the tester cell line by the titer of that same viral stock in the 
C127 control cell line. (In Tables 1-4 we report the mean "clonogenic efficiency" values calculated from at least three independent experiments.) Thus, those tester viruses that exhibit lower "clonogenic efficiency" than the control viruses (LXSN or ErbB4 in Tables 1 and 2; ErbB4 Q646C K751M in Table 3; ErbB4 Q646C K751M and ErbB4 Q646C EGFP in Table 4) are judged to inhibit clonogenic proliferation.

To make these judgments more obvious, we have also calculated "inhibition of clonogenic proliferation" for each tester virus by dividing the "clonogenic efficiency" of that tester virus by the "clonogenic efficiency" of the corresponding control virus and subtracting that result from $100 \%$. This value was calculated for each trial of at least three independent experiments. The mean and the standard error of these values are reported. The larger the mean value, the more the mutant behaves as an inhibitor of clonogenic proliferation.

\begin{tabular}{|l|l|l|l|}
\hline Cell Line & Retrovirus & $\begin{array}{l}\text { Clonogenic } \\
\text { Efficiency } \\
\text { (Relative to C127 } \\
\text { Cells })\end{array}$ & $\begin{array}{l}\text { Inhibition of Clono- } \\
\text { genic Proliferation } \\
\text { (Relative to ErbB4 } \\
\text { Q646C K751M) }\end{array}$ \\
\hline $\begin{array}{l}\text { DU-145 } \\
(\mathrm{n}=4)\end{array}$ & $\begin{array}{l}\text { ErbB4 Q646C } \\
\text { K751M }\end{array}$ & $7.4 \%$ & N/A \\
\cline { 2 - 4 } & ErbB4 Q646C & $0.3 \%$ & $95 \pm 2 \%$ \\
\cline { 2 - 4 } & $\begin{array}{l}\text { ErbB4 Q646C } \\
\text { EGFP }\end{array}$ & $6.0 \%$ & $15 \pm 9 \%$ \\
\cline { 2 - 4 } & $\begin{array}{l}\text { ErbB4 Q646C } \\
\text { EGFP-TVV }\end{array}$ & $1.2 \%$ & $83 \pm 4 \%$ \\
\hline \multirow{5}{*}{$\begin{array}{l}\text { PC-3 } \\
\text { n=3 })\end{array}$} & $\begin{array}{l}\text { ErbB4 Q646C } \\
\text { K751M }\end{array}$ & $12.0 \%$ & N/A \\
\cline { 2 - 4 } & ErbB4 Q646C & $1.4 \%$ & $90 \pm 3 \%$ \\
\cline { 2 - 4 } & $\begin{array}{l}\text { ErbB4 Q646C } \\
\text { EGFP }\end{array}$ & $10.9 \%$ & $15 \pm 8 \%$ \\
\cline { 2 - 4 } & $\begin{array}{l}\text { ErbB4 Q646C } \\
\text { EGFP-TVV }\end{array}$ & $1.1 \%$ & $89 \pm 3 \%$ \\
\hline
\end{tabular}

Table 3: Adding a carboxyl-terminal Enhanced Green Fluorescent Protein (EGFP) tag to the constitutively-active ErbB4 Q646C mutant disrupts its tumor suppressor activity, but this deficit is rescued by adding a carboxylterminal Thr-Val-Val (TVV) sequence.

\section{ErbB4 subcellular localization}

Various pLXSN-ErbB4-EGFP constructs (200 ng, uncut plasmid) were transiently transfected into the PC-3 human prostate tumor cell line using TurboFectin 8.0 (Origene). Cells were incubated for 48 hours, after which they were stained with Hoescht 33342 (Sigma - for DNA) and MitoTracker Red CMXRos (Molecular Probes - for mitochondria). Cells were imaged using a Radiance 2100 MP Rainbow (BioRad) laser scanning confocal unit attached to a TE2000 inverted microscope with a $60 x$ oil-immersion objective lens (Nikon). The MitoTracker Red (red images) was imaged following excitation at $543 \mathrm{~nm}$ using a green HeNe laser; the fluorescence emission greater than $560 \mathrm{~nm}$ was collected. EGFP (green images) was imaged following excitation with the $488 \mathrm{~nm}$ line of the 4-line Argon laser; the fluorescence emission was collected using a 500LP/550SP filter combination. Hoescht 33342 (blue images) was imaged following excitation at $350 \mathrm{~nm}$ using a Mai Tai laser (Spectra Physics); the emission between 420 and $480 \mathrm{~nm}$ was collected. We photographed multiple

\begin{tabular}{|c|c|c|c|}
\hline Cell Line & Retrovirus & $\begin{array}{r}\text { Clonogenic } \\
\text { Efficiency } \\
\text { (Relative to C127 } \\
\text { Cells) }\end{array}$ & $\begin{array}{r}\text { Inhibition of Clono- } \\
\text { genic Proliferation } \\
\text { (Relative to ErbB4 } \\
\text { Q646C K751M) }\end{array}$ \\
\hline \multirow[t]{10}{*}{$\begin{array}{r}\mathrm{DU}-145 \\
(\mathrm{n}=5)\end{array}$} & $\begin{array}{r}\text { ErbB4 Q646C } \\
\text { K751M }\end{array}$ & $4.6 \%$ & N/A \\
\hline & $\begin{array}{r}\text { ErbB4 Q646C } \\
\text { EGFP }\end{array}$ & $5.5 \%$ & None \\
\hline & $\begin{array}{r}\text { ErbB4 Q646C } \\
\text { EGFP-TVV }\end{array}$ & $0.8 \%$ & $82 \pm 2 \%$ \\
\hline & $\begin{array}{r}\text { ErbB4 Q646C } \\
\text { K751M EGFP- } \\
\text { TVV }\end{array}$ & $12.8 \%$ & None \\
\hline & $\begin{array}{r}\text { ErbB4 Q646C } \\
\text { V673I EGFP- } \\
\text { TVV }\end{array}$ & $5.2 \%$ & None \\
\hline & $\begin{array}{r}\text { ErbB4 Q646C } \\
\text { LL783/4AA } \\
\text { EGFP-TVV }\end{array}$ & $8.9 \%$ & None \\
\hline & $\begin{array}{r}\text { ErbB4 Q646C } \\
\text { LL867/8AA } \\
\text { EGFP-TVV }\end{array}$ & $0.4 \%$ & $92 \pm 2 \%$ \\
\hline & $\begin{array}{r}\text { ErbB4 Q646C } \\
\text { L985A EGFP- } \\
\text { TVV }\end{array}$ & $2.8 \%$ & $35 \pm 10 \%$ \\
\hline & $\begin{array}{r}\text { ErbB4 Q646C } \\
\text { D990A EGFP- } \\
\text { TVV }\end{array}$ & $1.4 \%$ & $69 \pm 4 \%$ \\
\hline & $\begin{array}{r}\text { ErbB4 Q646C } \\
\text { Y1056F EGFP- } \\
\text { TVV }\end{array}$ & $9.7 \%$ & None \\
\hline \multirow[t]{10}{*}{$\begin{array}{l}\mathrm{PC}-3 \\
(\mathrm{n}=5)\end{array}$} & $\begin{array}{r}\text { ErbB4 Q646C } \\
\text { K751M } \\
\end{array}$ & $15.7 \%$ & N/A \\
\hline & $\begin{array}{r}\text { ErbB4 Q646C } \\
\text { EGFP }\end{array}$ & $24.9 \%$ & None \\
\hline & $\begin{array}{r}\text { ErbB4 Q646C } \\
\text { EGFP-TVV } \\
\end{array}$ & $2.0 \%$ & $89 \pm 3 \%$ \\
\hline & $\begin{array}{r}\text { ErbB4 Q646C } \\
\text { K751M EGFP- } \\
\text { TVV }\end{array}$ & $22.9 \%$ & None \\
\hline & $\begin{array}{r}\text { ErbB4 Q646C } \\
\text { V673I EGFP- } \\
\text { TVV }\end{array}$ & $8.9 \%$ & $28 \pm 17 \%$ \\
\hline & $\begin{array}{r}\text { ErbB4 Q646C } \\
\text { LL783/4AA } \\
\text { EGFP-TVV } \\
\end{array}$ & $26.1 \%$ & None \\
\hline & $\begin{array}{r}\text { ErbB4 Q646C } \\
\text { LL867/8AA } \\
\text { EGFP-TVV }\end{array}$ & $3.8 \%$ & $80 \pm 3 \%$ \\
\hline & $\begin{array}{r}\text { ErbB4 Q646C } \\
\text { L985A EGFP- } \\
\text { TVV }\end{array}$ & $8.5 \%$ & $49 \pm 6 \%$ \\
\hline & $\begin{array}{r}\text { ErbB4 Q646C } \\
\text { D990A EGFP- } \\
\text { TVV }\end{array}$ & $3.3 \%$ & $78 \pm 3 \%$ \\
\hline & $\begin{array}{r}\text { ErbB4 Q646C } \\
\text { Y1056F EGFP- } \\
\text { TVV }\end{array}$ & $23.4 \%$ & None \\
\hline
\end{tabular}

Table 4: The K751M, V673I, LL783/4AA, and Y1056F mutations markedly disrupt the tumor suppressor activity of the constitutively-active ErbB4 Q646C EGFP-TVV construct in the DU-145 and PC-3 human prostate tumor cell lines. 
randomly-selected GFP-positive cells per transfected plasmid per experiment. Photomicrographs shown are representative of results from at least three independent experiments.

\section{Results}

The K751M, V673I, LL783/4AA, and L985A mutations profoundly disrupt the tumor suppressor activity of the constitutively-active ErbB4 Q646C mutant. We have previously constructed a constitutively-dimerized and -active form of the ErbB4 receptor tyrosine kinase by substituting a cysteine residue for Gln646 of the ErbB4 extracellular juxtamembrane region (ErbB4 Q646C mutant - Figure 1). The ErbB4 Q646C mutant inhibits clonogenic proliferation of several human cell lines, including the DU-145 and PC-3 human prostate tumor cell lines $[43,44]$, the SKBR3 and MCF7 human breast tumor cell lines, the MCF10A human breast epithelial cell line [42], and the CaPan-1, HPAC, MIA-PaCa2, and PANC-1 human pancreatic tumor cell lines [40]. This tumor suppressor activity displayed by the ErbB4 Q646C mutant requires ErbB4 tyrosine kinase activity and the carboxyl-terminal, cytoplasmic Tyr1056 residue $[42,44]$. However, it is not known whether other putative ErbB4 functional motifs are required for the tumor suppressor activity of the ErbB4 Q646C mutant.

In agreement with these published results, here we show that, relative to control constructs, the ErbB4 Q646C mutant inhibits clonogenic proliferation of (or displays tumor suppressor activity in) MCF7 human breast tumor cells (Figure 2a and 2b, Table 1) and of MCF10A human breast epithelial cells (Figure $2 \mathrm{c}$, Table 2). Also consistent with published data [42, 44], the K751M mutation, which disrupts tyrosine kinase activity, disrupts the tumor suppressor activity of the ErbB4 Q646C mutant in the MCF7 and MCF10A cells (Figure 2, Table 1 and Table 2). The V673I, LL783/4AA, and L985A mutations presumably abrogate ErbB4 cleavage by gamma-secretase [10], prevent ErbB4 interactions with steroid hormone receptors [13], and prevent ErbB4 interactions with $\mathrm{Bcl}$ family proteins [53], respectively. These mutations disrupt inhibition of clonogenic proliferation by (disrupt tumor suppressor activity of) the ErbB4 Q646C mutant (Figure 2, Table 1, and Table 2), thereby suggesting the importance of these putative motifs for the tumor suppressor activity of the constitutively-active ErbB4 Q646C mutant.

The V673I, LL783/4AA, and L985A mutations do not markedly alter the expression or tyrosine phosphorylation of the constitutively-active ErbB4-Q646C construct. Another explanation for the potential disruption of ErbB4 tumor suppressor activity by the V673I, LL783/4AA, and L985A mutations is that these mutations may abrogate ErbB4 expression and/ or tyrosine phosphorylation. However, the V673I (Figure 3a), LL783/4AA (Figure 3a), and L985A (Figure 3b) mutations do not disrupt the expression or tyrosine phosphorylation of the constitutively-active ErbB4 Q646C mutant. Therefore, the abrogation of tumor suppressor activity of the constitutivelyactive ErbB4 Q646C mutant by the V673I, LL783/4AA, and L985A mutants appears to be due to the disruption of the gamma-secretase cleavage site, the amino-terminal steroid hormone binding motif, and the $\mathrm{BH} 3$ motif, respectively.
A.

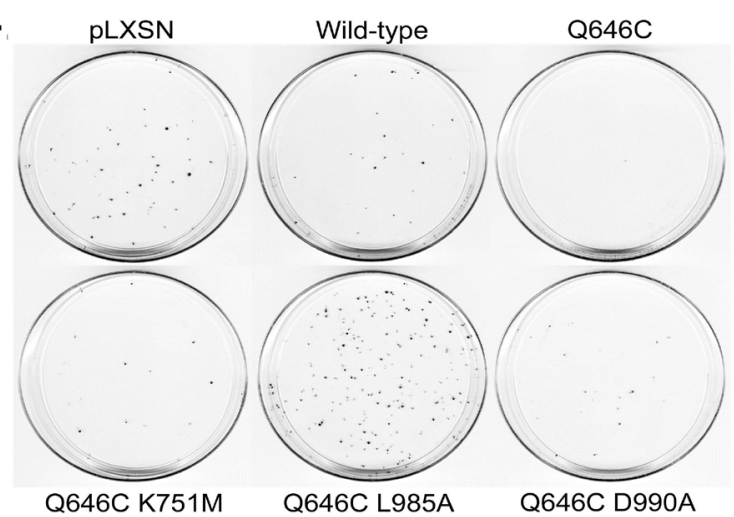

B.

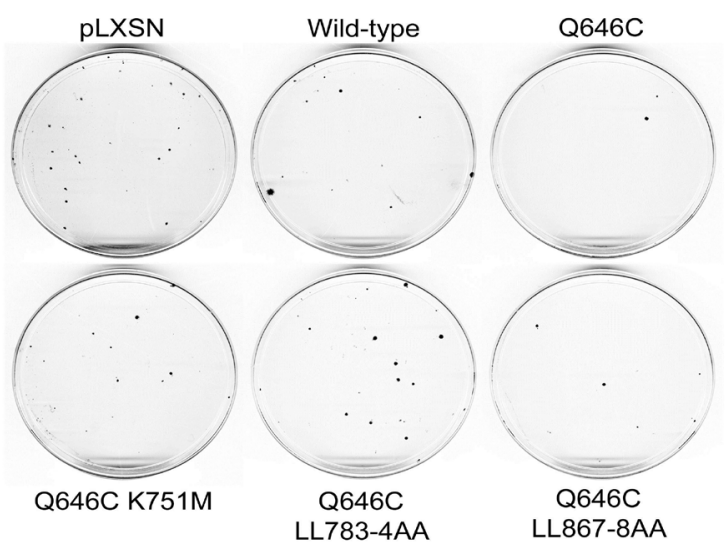

C.
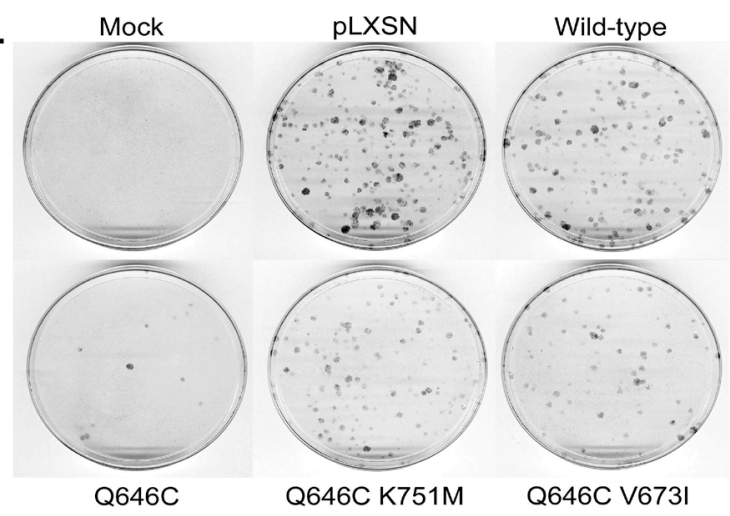

Figure 2. K751M, V673I, LL783/4AA, and L985A mutations profoundly disrupt the tumor suppressor activity of the constitutively-activeErbB4 Q646C construct in MCF7 and MCF10A cell lines. (a,b) MCF7 cells were infected with recombinant retroviruses based on the pLXSN vector as indicated. Infected cells were selected using G418 and colonies of infected, drug-resistant cells were stained using Giemsa and photographed. Images are representative of four independent experiments. The number of colonies was counted and the effects of the various constructs on clonogenic proliferation of MCF7 cells were analyzed as indicated elsewhere and reported in Table 1. (c) MCF10A cells were infected with recombinant retroviruses based on the $\mathrm{PLXSN}$ vector as indicated. Infected cells were selected using G418 and colonies of infected, drug-resistant cells were stained using Giemsa and photographed. Images are representative of at least three independent experiments. The number of colonies was counted and the effects of the various constructs on clonogenic proliferation of MCF10A cells were analyzed as indicated elsewhere and reported in Table 2.

A C-terminal EGFP tag abrogates the tumor suppressor activity of the ErbB4 Q646C mutant, but is rescued by adding a TVV tag. The data shown thus far suggests that the tumor suppressor activity of the constitutively-active ErbB4 Q646C mutant 
A.
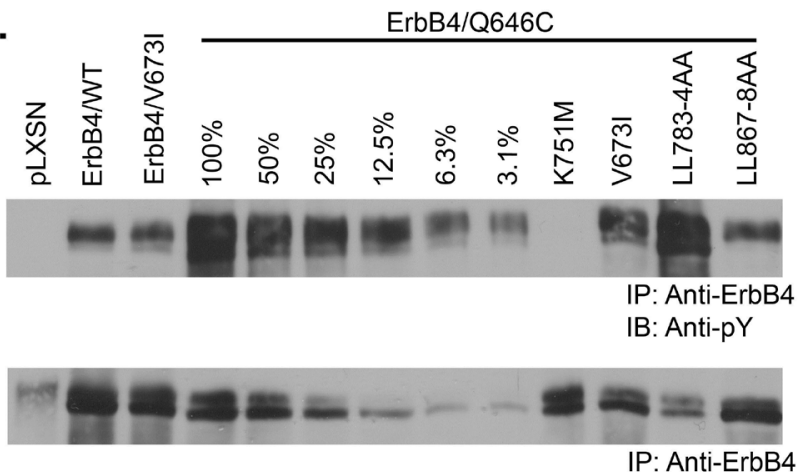

IB: Anti-ErbB4

B.

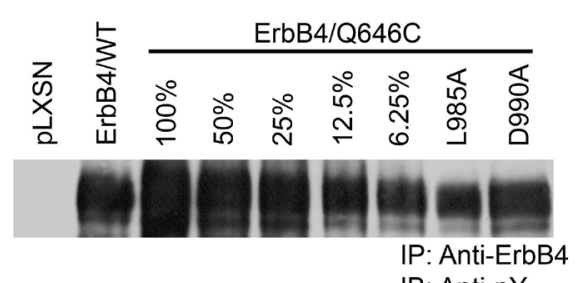

IB: Anti-pY

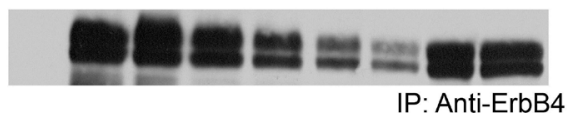

IB: Anti-ErbB4

Figure 3: The V673I, LL783/4AA, and L985A mutations do not markedly alter the expression or tyrosine phosphorylation of the constitutively-active ErbB4 Q646C construct. The expression and tyrosine phosphorylation of ErbB4 mutants expressed in $\Psi^{2}$ cell lines generated in the course of the experiments described in Figure 2 were analyzed by ErbB4 immunoprecipitation of $\Psi^{2}$ cell lysates and ErbB4 or anti-phosphotyrosine immunoblotting as described previously $[40,41]$. These experiments were performed using $1 \mathrm{mg}$ of lysate unless otherwise noted (100\% indicates that $1 \mathrm{mg}$ of lysate was used, $50 \%$ indicates that $0.5 \mathrm{mg}$ of lysate was used, $25 \%$ indicates that $0.25 \mathrm{mg}$ of lysate was used, and so on). Images are representative of three independent experiments.

is dependent on cleavage by gamma-secretase and on interactions of ErbB4 with steroid hormone receptors and Bcl family proteins. Thus, we postulated that the tumor suppressor activity of the ErbB4 Q646C mutant might be dependent on subcellular trafficking of ErbB4 away from the plasma membrane and to the cytoplasm, the nucleus, and the mitochondria. To test this hypothesis, we introduced the Q646C mutation into an ErbB4 construct that possesses an Enhanced Green Fluorescent Protein (EGFP) tag at the carboxyl-terminus of ErbB4 (Figure 4). Unfortunately, unlike the parental ErbB4 Q646C construct, the resulting ErbB4 Q646C EGFP construct fails to effectively inhibit clonogenic proliferation of PC-3 (Figure 5a) or DU-145 (Figure 5b) human prostate tumor cell lines (Table 3). The carboxyl-terminal Thr-Val-Val (TVV) motif of ErbB4 regulates ErbB4 trafficking, presumably by interacting with proteins that possess PDZ domains $[17,18]$. Therefore we postulated that adding a TVV sequence to the carboxyl terminus of the ErbB4 Q646C EGFP construct would rescue its tumor suppressor activity. Indeed, the ErbB4 Q646C EGFP-TVV construct inhibited clonogenic proliferation of PC-3 (Figure $5 \mathrm{a}$ ) or DU-145 (Figure 5b) human prostate tumor cell lines to almost the same extent as the untagged ErbB4 Q646C construct (Table 3). Therefore, the ErbB4 Q646C EGFP-TVV construct is a tool that can be used to assess the effects of ErbB4 functional motif mutations on the subcellular localization and tumor suppressor activity of the ErbB4 Q646C mutant.

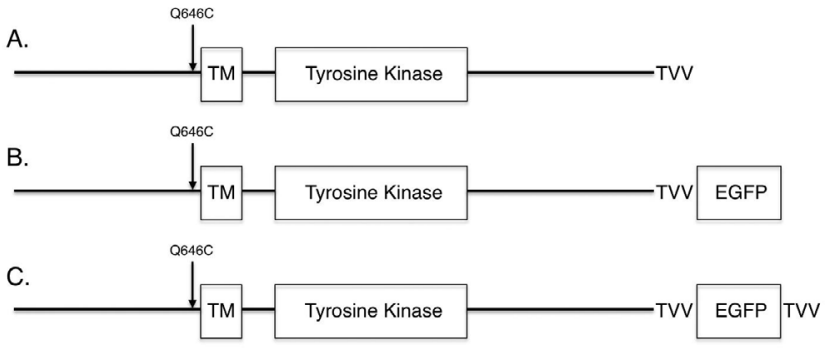

Figure 4: An Enhanced Green Fluorescent Protein (EGFP) tag and the amino acid sequence Thr-Val-Val (TVV) have been added to the carboxyl terminus of the ErbB4 Q646C mutant. Standard subcloning techniques were used to move the EGFP sequence from the ErbB4-EGFP construct [10] to the ErbB4 Q646C construct [41], thereby generating the ErbB4 Q646C EGFP construct. Standard site-directed mutagenesis techniques were used to add the Thr-ValVal (TVV) sequence to the extreme carboxyl terminus of the ErbB4 Q646C EGFP construct, thereby generating the ErbB4 Q646C EGFP-TVV construct.
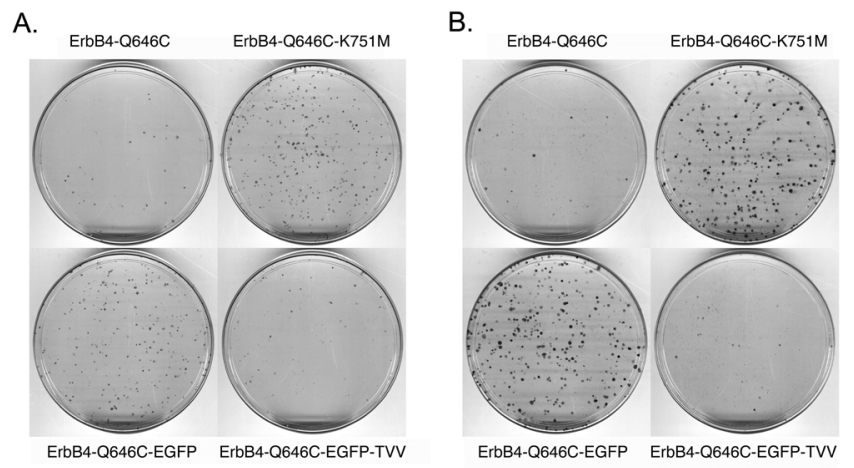

Figure 5: Adding a carboxyl-terminal Enhanced Green Fluorescent Protein (EGFP) tag to the constitutively-active ErbB4 Q646C mutant disrupts its tumor suppressor activity, but this deficit is rescued by adding a carboxyl-terminal Thr-Val-Val (TVV) sequence. (a) PC-3 cells were infected with recombinant retroviruses based on the pLXSN-ErbB4 vector as indicated. Infected cells were selected using G418 and colonies of infected, drug-resistant cells were stained using Giemsa and photographed. Images are representative of three independent experiments. The number of colonies was counted and the effects of the various constructs on clonogenic proliferation of PC-3 cells were analyzed as indicated elsewhere and reported in Table 3. (b) DU-145 cells were infected with recombinant retroviruses based on the pLXSN vector as indicated. Infected cells were selected using G418 and colonies of infected, drug-resistant cells were stained using Giemsa and photographed. Images are representative of four independent experiments. The colonies were counted and the effects of the various constructs on clonogenic proliferation of DU145 cells were analyzed as indicated elsewhere and reported in Table 3.

The K751M, V673I, LL783/4AA, L985A, and Y1056F mutations disrupt the tumor suppressor activity of the ErbB4 Q646C EGFP-TVV construct. In results that are similar to those shown elsewhere in this report, mutations that disrupt ErbB4 kinase activity (K751M), ErbB4 cleavage by gamma-secretase (V673I), ErbB4 interactions with steroid hormone receptors (LL783/4AA), ErbB4 interactions with Bcl family proteins (L985A), and ErbB4 phosphorylation at Tyr1056 (Y1056F) all disrupt inhibition of clonogenic proliferation of (or disrupt tumor suppressor activity in) the PC-3 (Figure 6) and DU145 (Figure 7) human prostate tumor cell lines (Table 4). Thus, ErbB4 kinase activity, ErbB4 cleavage by gamma-secretase, ErbB4 interactions with steroid hormone receptors, ErbB4 interactions with $\mathrm{Bcl}$ family proteins, and interactions with ef- 
fectors via binding to ErbB4 phospho-Tyr1056 all appear to be critical for the tumor suppressor activity of the constitutivelyactive ErbB4 Q646C EGFP-TVV construct.
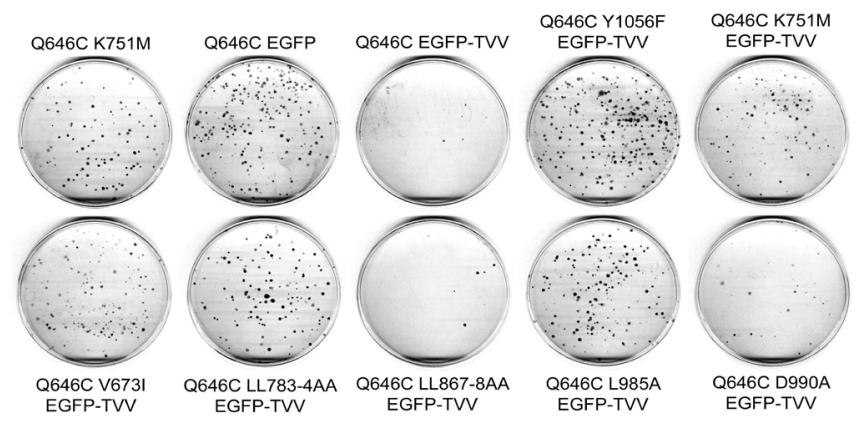

Figure 6: The K751M, V673I, LL783/4AA, and Y1056F mutations markedly disrupt the tumor suppressor activity of the constitutively-active ErbB4 Q646C EGFP-TVV construct in thePC-3 human prostate tumor cell line. PC-3 cells were infected with recombinant retroviruses based on the pLXSN-ErbB4 vector as indicated. Infected cells were selected using G418 and colonies of infected, drug-resistant cells were stained using Giemsa and photographed. Images are representative of five independent experiments. The number of colonies was counted and the effects of the various constructs on clonogenic proliferation of PC-3 cells were analyzed as indicated elsewhere and reported in Table 4.
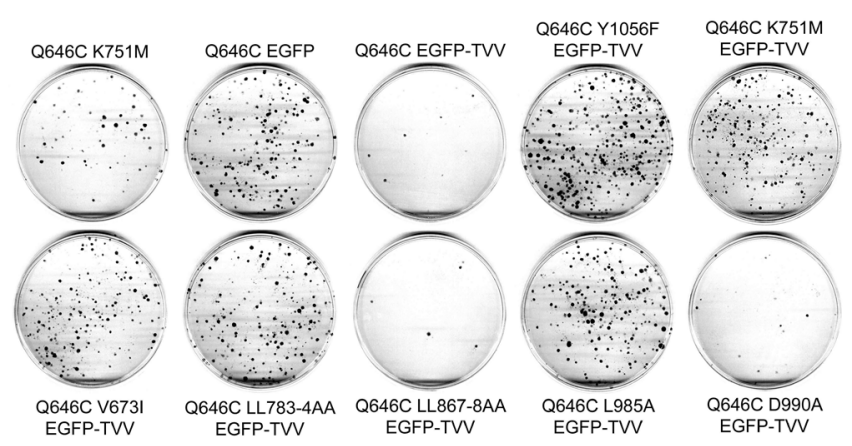

Figure 7: The K751M, V673I, LL783/4AA, and Y1056F mutations markedly disrupt the tumor suppressor activity of the constitutively-active ErbB4 Q646C EGFP-TVV construct in the DU-145 human prostate tumor cell line. DU-145 cells were infected with recombinant retroviruses based on the pLXSN vector as indicated. Infected cells were selected using G418 and colonies of infected, drug-resistant cells were stained using Giemsa and photographed. Images are representative of five independent experiments. The colonies were counted and the effects of the various constructs on clonogenic proliferation of DU145 cells were analyzed as indicated elsewhere and reported in Table 4.

The K751M, V673I, and Y1056F mutations disrupt the subcellular localization of the ErbB4 Q646C EGFP-TVV construct. Next, we used confocal microscopy to assess whether ErbB4 mutations that disrupt the tumor suppressor activity of the constitutively-active ErbB4 Q646C EGFP-TVV construct also alter its subcellular localization (Figure 8). Imaging of the EGFP tag reveals that the positive control ErbB4 Q646C EGFP-TVV construct displays plasma membrane expression as well as both diffuse and punctate expression throughout the cytoplasm (Figure 8), suggesting that ErbB4 is cleaved and trafficked away from the plasma membrane. In contrast, the K751M, V673I, and Y1056F mutants predominantly exhibit plasma membrane expression, only modest diffuse cytoplasmic expression, and little punctate cytoplasmic expression (Figure 8). This suggests that the absence of tumor suppressor activity displayed by these mutants may be a result of ErbB4 mislocalization. The L985A and LL783/4AA mutations, which disrupt the tumor suppressor activity of the ErbB4 Q646C EGFP-TVV construct, retain cytoplasmic expression (data not shown), suggesting that ErbB4 trafficking to the cytoplasm is necessary, but not sufficient, for the tumor suppressor activity of the ErbB4 Q646C EGFP-TVV construct. Inadequate expression or resolution or repression of an active translational event may account for the failure to observe nuclear or mitochondrial expression of the ErbB4 Q646C EGFP-TVV construct. However, we note that disrupting the $\mathrm{BH} 3$ domain or the amino-terminal LXXLL motif of ErbB4 abrogates the tumor suppressor activity of the ErbB4 Q646C mutant, suggesting that ErbB4 targeting to the nucleus and mitochondria is important for the tumor suppressor activity of the ErbB4 Q646C mutant. Because the ErbB4 Q646C EGFP-TVV construct retains tumor suppressor activity, it is reasonable to postulate that it too undergoes translocation to the nucleus and mitochondria and that inadequate expression or resolution accounts for the failure to observe this translocation.
A.

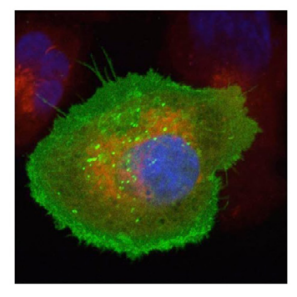

Q646C EGFP-TVV

\section{C.}

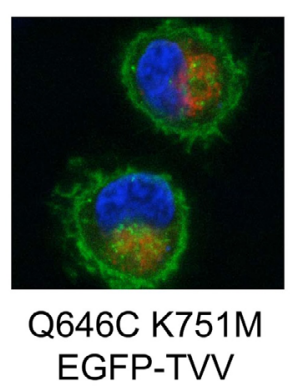

B.

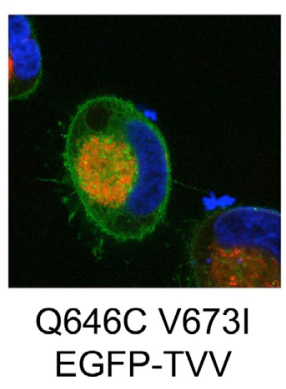

D.

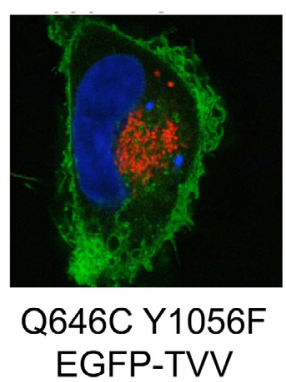

Figure 8: The K751M, V673I, and Y1056F mutations disrupt the subcellular localization of the ErbB4 Q646C EGFP-TVV construct. The various ErbB4 Q646C EGFP-TVV constructs were transiently transfected into the PC-3 human prostate tumor cell line, after which the cells were stained with Hoescht 33342 (for DNA - blue) or MitoTracker Red CMXRos (for mitochondria red). Cells were imaged by laser scanning confocal microscopy; the EGFPtagged proteins appear as green in these images. We photographed multiple randomly-selected, EGFP-positive cells per transfected plasmid per experiment. Images are representative of at least three independent experiments.

\section{Discussion}

Multiple functional motifs are required for the tumor suppressor activity of the constitutively-active ErbB4 Q646C mutant. Protein sequence and functional analyses suggest that ErbB4 possesses multiple functional motifs that are responsible for coupling ErbB4 to signaling effectors and biological responses (Figure 1). Here we have used mutations that target these putative functional motifs to demonstrate that several of these putative motifs are required for the tumor suppressor activity of the constitutively-active ErbB4 Q646C mutant. 
These results suggest that ErbB4 kinase activity and ErbB4 cleavage by gamma-secretase are both required for the tumor suppressor activity of the constitutively-active ErbB4 Q646C mutant. Likewise, ErbB4 interactions with a steroid hormone receptor(s) and with a Bcl family protein(s) also appear to be required for the tumor suppressor activity of the constitutively-active ErbB4 Q646C mutant. (These interactions appear to be site specific, as the LL867/8AA and D990A mutants retain at least some tumor suppressor activity.) Finally, the interaction between the ErbB4 phosphorylation site at Tyr1056 and an effector protein(s) appears to be required for the tumor suppressor activity of the constitutively-active ErbB4 Q646C mutant.

Alterations in ErbB4 subcellular localization may contribute to changes in ErbB4 function. Localization data presented here suggest that the ErbB4 motifs required for the tumor suppressor activity of the constitutively-active ErbB4 Q646C mutant may couple to this tumor suppressor activity in part by regulating ErbB4 cleavage and intracellular trafficking. This allows us to postulate a model that integrates ErbB4 trafficking, ErbB4 binding to effector proteins, and ErbB4 signaling activity. For example, the V673I mutation is expected to block ErbB4 cleavage by gamma-secretase, thereby preventing trafficking of the ErbB4 cytoplasmic domains away from the plasma membrane and to the cytoplasm, mitochondria, or nucleus. Similarly, the LL783/4AA mutation is expected to block interactions between ErbB4 and steroid hormone receptors, and thereby prevent steroid hormone receptors from directing ErbB4 to the nucleus. Finally, the L985A mutation is expected to block interactions between ErbB4 and Bcl family proteins, and thereby prevent $\mathrm{Bcl}$ family proteins from directing ErbB4 to the mitochondria.

It is not surprising that targeting the canonical site of ErbB4 binding (at ErbB4 phospho-Tyr1056) to the regulatory subunit of the PI3 kinase (through either the Y1056F or K751M mutations) disrupts the tumor suppressor activity of the constitutively-active ErbB4 Q646C mutant; the Y1056F or K751M mutations are predicted to prevent ErbB4 from coupling to PI3 kinase activity and to its pro-proliferative, anti-apoptotic effectors. However, it is somewhat surprising that the Y1056F mutant and the K751M mutant both fail to display cytoplasmic localization. This suggests that ErbB4 phosphorylation at Tyr1056 and consequent binding to the regulatory subunit of PI3 kinase is required for ErbB4 cleavage and/or trafficking away from the plasma membrane. This is somewhat at odds with the evidence suggesting that PI3 kinase functions primarily at the plasma membrane [58]. Indeed, another possibility may be that preventing phosphorylation at Tyr 1056 of ErbB4 alters ErbB4 binding to effectors that possess a WW domain(s) and that this change in effector binding alters the cleavage and/ or intracellular trafficking of ErbB4.

Heterodimerization of ErbB2 with ErbB4 may alter ErbB4 function by changing ErbB4 trafficking. We have previously demonstrated that in human pancreatic tumor cell lines in which the constitutively-active ErbB4 Q646C mutant functions as a tumor suppressor, wild-type ErbB4 appears to possess oncogenic activities [59]. We have attempted to rationalize this apparent paradox by demonstrating that the ErbB4 Q646C mutant functions as a homodimer in these cells, whereas wild-type ErbB4 functions as a heterodimer with EGFR or ErbB2. Specifically, we demonstrated that the oncogenic activities of wild-type ErbB4 require ErbB4 sites of tyrosine phosphorylation and EGFR or ErbB2 kinase activity, but not ErbB4 kinase activity. We postulated that EGFR or ErbB2 phosphorylates a set of ErbB4 tyrosine residues that is distinct from the set that are phosphorylated by ErbB4 itself [59]. Given the ErbB4 localization data shown in the work at hand, we now postulate that ErbB4 trafficking following heterodimerization with EGFR or ErbB2 may be quite different from ErbB4 trafficking resulting from ErbB4 homodimerization. Furthermore, this difference in trafficking may account for the functional differences between ErbB4 homodimers and ErbB2/ErbB4 or EGFR/ErbB4 heterodimers. Consequently, additional experimentation is warranted to further elucidate the roles that intracellular trafficking plays in regulating ErbB4 function, particularly in the context of ErbB4 homodimers and heterodimers.

\section{Acknowledgments}

We gratefully acknowledge the support of the American Society for Microbiology (INB), the Purdue University MARCAIM program (INB), the HHMI Minority Undergraduate Research Program (INB), the Purdue University Doctoral Fellowship Program (INB), the Purdue University Andrews Fellowship Program (RMG), the US Army Breast Cancer Research Program (DAMD17-00-1-0415, DAMD17-00-1-0416 to DJR), the US Army Prostate Cancer Research Program (DAMD17-02-1-0130 to DJR), the National Cancer Institute (R01CA114209 to DJR, P30CA023168), the American Cancer Society (IRG 58-006-40), the Showalter Trust, the Purdue University Center for Cancer Research, the Indiana Elks Foundation, the Carroll County (Indiana) Cancer Association, the Purdue University Confocal Microscopy Core, the Purdue University College of Pharmacy, the Auburn University Harrison School of Pharmacy, and the Auburn University Internal Grants Program.

\section{References}

1. Riese DJ 2nd, Stern DF (1998) Specificity within the EGF family/ErbB receptor family signaling network. Bioessays 20: 41-48.

2. Yarden Y, Sliwkowski MX (2001) Untangling the ErbB signalling network. Nat Rev Mol Cell Biol 2: 127-137.

3. Riese DJ 2nd, Gallo RM, Settleman J (2007) Mutational activation of ErbB family receptor tyrosine kinases: insights into mechanisms of signal transduction and tumorigenesis. Bioessays 29: 558-565.

4. Wilson KJ, Gilmore JL, Foley J, Lemmon MA, Riese DJ 2nd, et al. (2009) Functional selectivity of EGF family peptide growth factors: implications for cancer. Pharmacol Ther 122: 1-8.

5. Elenius K, Corfas G, Paul S, Choi CJ, Rio C, et al. (1997) A novel juxtamembrane domain isoform of HER4/ErbB4. Isoform-specific tissue distribution and differential processing in response to phorbol ester. J Biol Chem 272: 26761-26768.

6. Gilbertson R, Hernan R, Pietsch T, Pinto L, Scotting P, et al. (2001) Novel ERBB4 juxtamembrane splice variants are frequently expressed in childhood medulloblastoma. Genes Chromosomes Cancer 31: 288-294. 
7. Cheng QC, Tikhomirov O, Zhou W, Carpenter G (2003) Ectodomain cleavage of ErbB-4: characterization of the cleavage site and $\mathrm{m} 80$ fragment. J Biol Chem 278: 38421-38427.

8. Ni CY, Murphy MP, Golde TE, Carpenter G (2001) gamma -Secretase cleavage and nuclear localization of ErbB-4 receptor tyrosine kinase. Science 294: 2179-2181.

9. Rio C, Buxbaum JD, Peschon JJ, Corfas G (2000) Tumor necrosis factoralpha-converting enzyme is required for cleavage of erbB4/HER4. J Biol Chem 275: 10379-10387.

10. Williams CC, Allison JG, Vidal GA, Burow ME, Beckman BS (2004) The ERBB4/HER4 receptor tyrosine kinase regulates gene expression by functioning as a STAT5A nuclear chaperone. J Cell Biol 167: 469-478.

11. Edwards DP (2000) The role of coactivators and corepressors in the biology and mechanism of action of steroid hormone receptors. J Mammary Gland Biol Neoplasia 5: 307-324.

12. Heery DM, Kalkhoven E, Hoare S, Parker MG (1997) A signature motif in transcriptional co-activators mediates binding to nuclear receptors. Nature 387: 733-736.

13. Savkur RS, Burris TP (2004) The coactivator LXXLL nuclear receptor recognition motif. J Pept Res 63: 207-212.

14. Naresh A, Long W, Vidal GA, Wimley WC, Marrero L, et al. (2006) The ERBB4/HER4 intracellular domain 4ICD is a BH3-only protein promoting apoptosis of breast cancer cells. Cancer Res 66: 6412-6420.

15. Komuro A, Nagai M, Navin NE, Sudol M (2003) WW domain-containing protein YAP associates with ErbB-4 and acts as a co-transcriptional activator for the carboxyl-terminal fragment of ErbB-4 that translocates to the nucleus. J Biol Chem 278: 33334-33341.

16. Omerovic J, Puggioni EM, Napoletano S, Visco V, Fraioli R, et al. (2004) Ligand-regulated association of ErbB-4 to the transcriptional co-activator YAP65 controls transcription at the nuclear level. Exp Cell Res 294: 469-479.

17. Hung AY, Sheng M (2002) PDZ domains: structural modules for protein complex assembly. J Biol Chem 277: 5699-5702.

18. Ni CY, Yuan H, Carpenter G (2003) Role of the ErbB-4 carboxyl terminus in gamma-secretase cleavage. J Biol Chem 278: 4561-4565.

19. Arteaga CL (2006) EGF receptor mutations in lung cancer: from humans to mice and maybe back to humans. Cancer Cell 9: 421-423.

20. Arteaga CL, Moulder SL, Yakes FM (2002) HER (erbB) tyrosine kinase inhibitors in the treatment of breast cancer. Semin Oncol 29: 4-10.

21. Hynes NE, Stern DF (1994) The biology of erbB-2/neu/HER-2 and its role in cancer. Biochim Biophys Acta 1198: 165-184.

22. Ji H, Sharpless NE, Wong KK (2006) EGFR targeted therapy: View from biological standpoint. Cell Cycle 5: 2072-2076.

23. Roskoski R Jr (2004) The ErbB/HER receptor protein-tyrosine kinases and cancer. Biochem Biophys Res Commun 319: 1-11.

24. Bacus SS, Chin D, Yarden Y, Zelnick CR, Stern DF (1996) Type 1 receptor tyrosine kinases are differentially phosphorylated in mammary carcinoma and differentially associated with steroid receptors. Am J Pathol 148: 549-558.

25. Barnes NL, Khavari S, Boland GP, Cramer A, Knox WF, et al. (2005) Absence of HER4 expression predicts recurrence of ductal carcinoma in situ of the breast. Clin Cancer Res 11: 2163-2168.

26. deFazio A, Chiew YE, Sini RL, Janes PW, Sutherland RL (2000) Expression of c-erbB receptors, heregulin and oestrogen receptor in human breast cell lines. Int J Cancer 87: 487-498.

27. Junttila TT, Sundvall M, Lundin M, Lundin J, Tanner M, et al. (2005) Cleavable ErbB4 isoform in estrogen receptor-regulated growth of breast cancer cells. Cancer Res 65:1384-1393.

28. Kew TY, Bell JA, Pinder SE, Denley H, Srinivasan R, et al. (2000) c-erbB-4 protein expression in human breast cancer. Br J Cancer 82(6): 1163-1170.
29. Lyne JC, Melhem MF, Finley GG, Wen D, Liu N, et al. (1997) Tissue expression of neu differentiation factor/heregulin and its receptor complex in prostate cancer and its biologic effects on prostate cancer cells in vitro. Cancer J Sci Am 3: 21-30.

30. Memon AA, Sorensen BS, Melgard P, Fokdal L, Thykjaer T, et al. (2004) Expression of HER3, HER4 and their ligand heregulin-4 is associated with better survival in bladder cancer patients. Br J Cancer 91: 2034-2041.

31. Robinson D, He F, Pretlow T, Kung HJ (1996) A tyrosine kinase profile of prostate carcinoma. Proc Natl Acad Sci U S A 93: 5958-5962.

32. Srinivasan R, Poulsom R, Hurst HC, Gullick WJ (1998) Expression of the c-erbB-4/HER4 protein and mRNA in normal human fetal and adult tissues and in a survey of nine solid tumour types. J Pathol,. 185: 236-245.

33. Thybusch-Bernhardt A, Beckmann S, Juhl H (2001) Comparative analysis of the EGF-receptor family in pancreatic cancer: expression of HER-4 correlates with a favourable tumor stage. Int J Surg Investig 2: 393-400.

34. Vidal GA, Clark DE, Marrero L, Jones FE (2007) A constitutively active ERBB4/HER4 allele with enhanced transcriptional coactivation and cell-killing activities. Oncogene 26: 462-466.

35. Vidal GA, Naresh A, Marrero L, Jones FE (2005) Presenilin-dependent gamma-secretase processing regulates multiple ERBB4/HER4 activities. J Biol Chem 280: 19777-19783.

36. Sundvall M, Iljin K, Kilpinen S, Sara H, Kallioniemi OP, et al. (2008) Role of ErbB4 in breast cancer. Journal of Mammary Gland Biology \& Neoplasia 13: $259-268$

37. Muraoka-Cook RS, Feng SM, Strunk KE, Earp HS (2008) ErbB4/HER4: role in mammary gland development, differentiation and growth inhibition. J Mammary Gland Biol Neoplasia 13: 235-246.

38. Veikkolainen V, Vaparanta K, Halkilahti K, Iljin K, Sundvall M, et al. (2011) Function of ERBB4 is determined by alternative splicing. Cell Cycle 10: $2647-2657$.

39. Gordon-Thomson C, Jones J, Mason RS, Moore GP (2005) ErbB receptors mediate both migratory and proliferative activities in human melanocytes and melanoma cells. Melanoma Res 15: 21-28.

40. Mill CP, Gettinger KL, Riese DJ 2nd (2011) Ligand stimulation of ErbB4 and a constitutively-active ErbB4 mutant result in different biological responses in human pancreatic tumor cell lines. Exp Cell Res 317:b392-404.

41. Penington DJ, Bryant I, Riese DJ 2nd (2002) Constitutively active ErbB4 and ErbB2 mutants exhibit distinct biological activities. Cell Growth Differ 13: $247-256$

42. Pitfield SE, Bryant I, Penington DJ, Park G, Riese DJ 2nd (2006) Phosphorylation of ErbB4 on tyrosine 1056 is critical for ErbB4 coupling to inhibition of colony formation by human mammary cell lines. Oncol Res 16: 179-293.

43. Williams EE, Trout LJ, Gallo RM, Pitfield SE, Bryant I, et al. (2003) A constitutively active ErbB4 mutant inhibits drug-resistant colony formation by the DU-145 and PC-3 human prostate tumor cell lines. Cancer Lett 192: 67-74.

44. Gallo RM, Bryant I, Fry R, Williams EE, Riese DJ (2006) Phosphorylation of ErbB4 on Tyr1056 is critical for inhibition of colony formation by prostate tumor cell lines. Biochem Biophys Res Commun 349: 372-382.

45. Jones FE(2008) HER4 intracellular domain (4ICD) activity in the developing mammary gland and breast cancer. J Mammary Gland Biol Neoplasia. 13: $247-258$

46. Naresh A, Long W, Vidal GA, Wimley WC, Marrero L et al. (2006) The ERBB4/HER4 intracellular domain 4ICD is a BH3-only protein promoting apoptosis of breast cancer cells. Cancer Res 66: 6412-6420.

47. Naresh A, Thor AD, Edgerton SM, Torkko KC, Kumar R, Jones FE, et al. (2008) The HER4/4ICD estrogen receptor coactivator and BH3-only protein is an effector of tamoxifen-induced apoptosis. Cancer Res 68: 6387-6395.

48. Rokicki J, Das PM, Giltnane JM, Wansbury O, Rimm DL, et al. (2010) The ERalpha coactivator, HER4/4ICD, regulates progesterone receptor expression in normal and malignant breast epithelium. Mol Cancer 9: 150. 
49. Thor AD, Edgerton SM, Jones FE (2009) Subcellular localization of the HER4 intracellular domain, 4ICD, identifies distinct prognostic outcomes for breast cancer patients. Am J Pathol 175: 1802-1809.

50. Zhu Y, Sullivan LL, Nair SS, Williams CC, Pandey AK, et al. (2006) Coregulation of estrogen receptor by ERBB4/HER4 establishes a growth-promoting autocrine signal in breast tumor cells. Cancer Res 66: 7991-7998.

51. Leptak C, Ramon y Cajal S, Kulke R, Horwitz BH, Riese DJ 2nd, et al. (1991) Tumorigenic transformation of murine keratinocytes by the E5 genes of bovine papillomavirus type 1 and human papillomavirus type 16. J Virol 65: 7078-7083

52. Riese DJ 2nd, van Raaij TM, Plowman GD, Andrews GC, Stern DF (1995) The cellular response to neuregulins is governed by complex interactions of the erbB receptor family. Mol Cell Biol 15: 5770-5776.

53. Kelekar A, Thompson CB (1998) Bcl-2-family proteins: the role of the BH3 domain in apoptosis. Trends Cell Biol 8: 324-330.

54. Wilson KJ, Mill CP, Gallo RM, Cameron EM, VanBrocklin H, et al. (2012) The Q43L mutant of neuregulin 2beta is a pan-ErbB receptor antagonist. Biochem J 443: 133-144.
55. Miller AD, Rosman GJ (1989) Improved retroviral vectors for gene transfer and expression. Biotechniques 7: 980-982, 984-986, 989-990.

56. Mann R, Mulligan RC, Baltimore D (1983) Construction of a retrovirus packaging mutant and its use to produce helper-free defective retrovirus. Cell 33: 153-159.

57. Miller AD, Buttimore C (1986) Redesign of retrovirus packaging cell lines to avoid recombination leading to helper virus production. Mol Cell Biol 6: 2895-2902.

58. Sheppard K, Kinross KM, Solomon B, Pearson RB, Phillips WA (2012) Targeting PI3 kinase/AKT/mTOR signaling in cancer. Critical Reviews in Oncogenesis 17: 69-95.

59. Mill CP, Zordan MD, Rothenberg SM, Settleman J, Leary JF, et al. (2011) ErbB2 Is Necessary for ErbB4 Ligands to Stimulate Oncogenic Activities in Models of Human Breast Cancer. Genes Cancer 2: 792-804. 